\title{
A favorable case where an experimental electron density analysis offers a lead for understanding a specific fluxional process observed in solution
}

\author{
Yannick Ortin, ${ }^{\dagger}$ Noël Lugan, ${ }^{* \dagger}$ Sébastien Pillet, ${ }^{\ddagger}$ Mohammed Souhassou, ${ }^{\ddagger}$ Claude \\ Lecomte, ${ }^{* \prime *}$ Karine Costuas, ${ }^{\S}$ and Jean-Yves Saillard ${ }^{* ` \S}$ \\ Laboratoire de Chimie de Cordination du CNRS, 205 route de Narbonne, 31077 Toulouse Cedex 4, \\ France, Laboratoire de Cristallographie et de Modélisation des Matériaux Minéraux et \\ Biologiques, Université Henri Poincaré, Faculté des Sciences,54506 Vandoeuvre-Lès-Nancy \\ Cedex, France, and Laboratoire de Chimie du Solide et Inorganique, Institut de Chimie \\ Moléculaire de Rennes, Université de Rennes 1, 35042 Rennes Cedex, France.
}

\section{SUPPLEMENTARY MATERIAL}

\section{Data collection and reduction}

Structure and charge density refinements

Table S1 Crystal data and structure refinement for compound 1.

Table S2 Atomic coordinates for compound $\mathbf{1}$ after the multipolar refinement.

Table S3 Anisotropic or isotropic displacement parameters for compound $\mathbf{1}$ after the multipolar refinement.

Table S4 Bond lengths $[\AA]$ and angles [ $\left.{ }^{\circ}\right]$ for compound 1 after the multipolar refinement.

Table S5 Multipolar refinement parameters for compound 1.

Table S6 Topological properties of the experimental and theoretical electron density at selected bond critical points.

Fig. S1 Residual electron density in 1 after the multipolar refinement in the plane C10/ Mn1/C3.

Fig. S2 Experimental deformation map for 1 in the plane C10/ Mn1/C3.

Fig. S3 Experimental static deformation map for 1 in the plane C10/ Mn1/C3.

Fig. S4 Total experimental ED $\rho(\mathrm{r})$ for 1 in the Mn1/C3/C10 plane.

Fig. S5 Total theoretical ED $\rho(\mathrm{r})$ for 1 in the Mn1/C3/C10 plane.

Fig. S6 Laplacian of the theoretical electron density for 1, in the C2/ Mn1/C3 plane.

Fig. S7 Laplacian of the experimental electron density for $\mathbf{1}$, in the C10/ Mn1/C3 plane.

Fig. S8 Laplacian of the theoretical electron density for 1, in the C10/ Mn1/C3 plane.

\footnotetext{
* To whom correspondence should be addressed. E-mail: lugan@lcc-toulouse.fr.

${ }^{\dagger}$ Laboratoire de Chimie de Coordination

‡ Université Henri Poincaré

§ Université de Rennes
} 
Data collection and reduction. A diamond-shaped black crystal of dimensions $0.22 \times 0.58$ x 0.66 $\mathrm{mm}^{3}$ was mounted on an Oxford Diffraction Xcalibur 4-circles diffractomer equipped with a Sapphire CCD detector and an Oxford Diffraction Cryojet $\mathrm{N}_{2}$ gas stream low-temperature device. Graphitemonochromatized $\operatorname{MoK} \alpha(\lambda=0.71069 \AA)$ radiation was used $(0.6 \mathrm{~mm}$ collimated) with a generator working at $50 \mathrm{kV}$ and $40 \mathrm{~mA}$. The crystal was cooled from $273 \mathrm{~K}$ to $100( \pm 1) \mathrm{K}$ with a mean temperature gradient of $-2 \mathrm{~K} / \mathrm{min}$. The detector was kept at $55 \mathrm{~mm}$ from the crystal and the data were collected by the $\omega$-scan method (frame width $\Delta \omega=0.75^{\circ}$ ) within the limits $0<2 \theta<136.7^{\circ}$ (max $\left.\sin \theta / \lambda=1.31 \AA^{-1}\right)$. Four different positions for the detector arm were used $\left(\theta_{d}=30^{\circ}, 13\right.$ sets of $\omega$ runs, 1989 frames, 10 s per frame; $\theta_{d}=55^{\circ}, 6$ sets of $\omega$ runs, 840 frames, 25 s per frame; $\theta_{d}=75^{\circ}$, 7 sets of $\omega$ runs, 1084 frames, 50 s per frame; $\theta_{d}=110^{\circ}, 5$ sets of $\omega$ runs, 630 frames, 50 s per frame). The program MOVIE was used to measure the shape of the crystal in view absorption correction. ${ }^{1}$ The frames were reduced using the CRYSALIS software package. ${ }^{1}$ Numerical absorption corrections based on the shape of the crystal were performed; the calculated minimum and maximum transmission coefficients are $T_{\min }=0.346$ and $T_{\max }=0.735 .^{2}$ The resulting 119756 reflections were merged in Laue group -1 with the use of the program SORTAV ${ }^{3}$ to give 35022 unique reflections up to a resolution of $1.31 \AA^{-1}$. This data set provided $69 \%$ of data $2.7^{\circ}<\theta<68.4^{\circ}\left(85 \%\right.$ of data $\left.2.7^{\circ}<\theta<52.8^{\circ}\right)$. Although the shape of the selected crystal was highly anisotropic, the low internal agreement indice $\left(\mathrm{R}_{\mathrm{int}}=0.021\right.$, $<$ redundacy $>=3.7$ (7.3 up to $\left.1.10 \AA^{-1}\right)$ ) support the good quality and consistency of the merged data set. Other crystallographic and data collection details are given in Table S1.

Structure and charge density refinements. In first step, the molecular geometry of the complex was established following a classical X-ray diffraction structure analysis. The crystal structure was solved in the P -1 space group using SIR92, ${ }^{4}$ then refined by full matrix least-squares on $\mathrm{F}^{2}$ using the SHELX $97^{5}$ implemented in the WINGX package. ${ }^{6}$ All non-hydrogen atoms were refined with anisotropic temperature factors using all reflections with $\mathrm{I}>2 \sigma(\mathrm{I})$ and $2 \theta_{\max }=50^{\circ}$ (weighting scheme : $w=1 /\left[\sigma^{2}\left(F o^{2}\right)+(0.0202 \mathrm{P})^{2}+0.80 \mathrm{P}\right]$, where $\left.\left.\mathrm{P}=\left(\operatorname{Max}\left(F o^{2}, 0\right)+2 F c^{2}\right) / 3\right)\right)$. The hydrogen atoms were

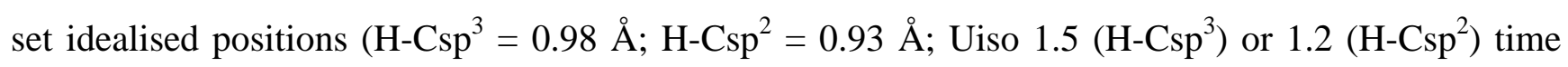
greater than the $\mathrm{U}_{e q}$ of the carbon atom to which the hydrogen atom is attached), and they were next refined as "riding” atoms. The refinement converged at $\mathrm{R}_{1}=0.0182, w \mathrm{R}_{2}=0.0472$, and $\mathrm{GOF}=1.147$ for 4721 observations (I>2 $\sigma(\mathrm{I}))$ and 380 variable parameters.

In the following refinements a multipole $\operatorname{model}^{7}$ was adopted to describe the deformation of $\rho(r)$ from a spherical distribution according to the Hansen and Coppens formalism as implemented in the MOPRO program. In this model, the electron density is described as a superposition of non spherical pseudoatoms (eq. 1):

$$
\rho(\vec{r})=\rho_{\text {core }}(r)+P_{v} \kappa^{3} \rho_{v a l}(\kappa r)+\sum_{l=0}^{l_{\max }} \kappa^{\prime 3} R_{l}\left(\kappa^{\prime} r\right) \sum_{m=0}^{+l} P_{l m} y_{l m \pm}(\vartheta, \varphi)
$$




$$
\text { where } R_{l}(r)=\frac{\xi_{l}^{n_{l}+3}}{\left(n_{l}+2\right) !} r^{n_{l}} e^{-\xi_{l} r}
$$

The first two terms of eq.(1)correspond, respectively, to spherically averaged Hartree-Fock core and valence electron density. $P_{\mathrm{v}}$ is the electron population of the valence shell. The third term expresses the aspherical part of the atomic electron density, projected on real spherical harmonics $y_{\operatorname{lm} \pm} . \kappa$ and $\kappa^{\prime}$ are contraction/expansion parameters.

The core and valence atomic spherical electron densities were constructed using Clementi HartreeFock atomic wave functions for ground-state isolated atoms expanded over Slater-type basis functions. ${ }^{8}$ In our model, the multipole expansion was truncated at the hexadecapole level $\left(l_{\max }=4\right)$ for the metal atoms, and at the octupole level $\left(l_{\max }=3\right)$ for the oxygen and carbon atoms. A H-C bond directed dipole $\left(l_{\max }=2\right)$ was introduced for the hydrogen atoms. The following coefficients $\left[\left(n_{1}=3,4\right.\right.$, 4, 4, $\left.\xi_{l}=7.40 \mathrm{bohr}^{-1}\right)$ for Co, $\left(n_{\mathrm{l}}=3,4,4,4, \xi_{l}=7.00 \mathrm{bohr}^{-1}\right)$ for Mn, $\left(n_{\mathrm{l}}=2,2,3, \xi_{l}=4.50 \mathrm{bohr}^{-1}\right)$ for O, $\left(n_{1}=2,2,3, \xi_{l}=3.14 \mathrm{bohr}^{-1}\right)$ for C, $\left(n_{1}=2, \xi_{l}=2.28 \mathrm{bohr}^{-1}\right)$ for H] were used for the Slater-type radial function $\mathrm{R}_{l}(\mathrm{r})$. The anomalous dispersion coefficients were taken from the International Tables. ${ }^{9}$

The starting atomic positions were taken from the SHELX97 refinement, and the C-H bond lengths were set to neutrons values $\left[\mathrm{H}-\mathrm{Csp}^{3}=1.093 \AA\right.$; $\left.\mathrm{H}^{3} \mathrm{Csp}^{2}=1.083 \AA\right]$ by shifting the hydrogen atoms along the $\mathrm{C}-\mathrm{H}$ directions. The positions and the thermal parameters were first refined using high resolution data only $\left(S>0.7 \AA^{-1}\right)$. The positions and the isotropic thermal parameters for the hydrogen atoms were allowed to vary in a restrained model as implemented in MOPRO $\left[\mathrm{H}-\mathrm{Csp}^{3}=1.093 \AA\right.$; H$\left.\mathrm{Csp}^{2}=1.083 \AA\right]$. Then the valence electron density was fitted using low resolution data only $(S<0.7$ $\AA^{-1}$ ) in successive cycles on $P_{\mathrm{v}}, \kappa, P_{\operatorname{lm} \pm}$ and $\kappa^{\prime}$ parameters, until convergence was reached. For metal centres, the multipoles were allowed to refine assuming a $3 \mathrm{~d}^{7}$ and $3 \mathrm{~d}^{5}$ valence configuration for Co and $\mathrm{Mn}$, respectively, the $4 \mathrm{~s}$ electrons being set in the core. Attempts to refine separately both $4 \mathrm{~s}$ and $3 \mathrm{~d}$ led to physically inconsistent coefficients. Constraints were applied to some multipole parameters and to some of the $\kappa / \kappa^{\prime}$ parameters. Indeed, the multipole parameters for $\mathrm{C}$ and $\mathrm{O}$ atoms were allowed to vary with respect to imposed symmetries within the functional groups [ $\mathrm{C}_{\infty \mathrm{v}}$ symmetry for the carbonyl ligands (except for $\mathrm{C}(2) \mathrm{O}(2)$ for which no constraints were applied), $\mathrm{C}_{\mathrm{s}}$ symmetry for methyl cyclopentadienyl ligand, $\mathrm{C}_{2}$ symmetry for the phenyl rings]. Three sets of $\kappa / \kappa$ ' for the three type of chemically different $\mathrm{O}$ atoms [Mn-CO, Co-CO, $\mathrm{R}(\mathrm{R}) \mathrm{C}=\mathrm{C}=\mathrm{O}$ ], eight sets of $\kappa / \kappa^{\prime}$ for the eight type of chemically different $\mathrm{C}$ atoms [Mn- $\mathrm{CO}, \mathrm{H}_{3} \mathrm{CC}_{5} \mathrm{H}_{4}, \mathrm{H}_{3} \mathrm{CC}_{5} \mathrm{H}_{4}, \mathrm{Co}-\mathrm{CO}, \mathrm{R}\left(\mathrm{R}{ }^{\prime}\right) C=\mathrm{C}=\mathrm{O}, \mathrm{R}\left(\mathrm{R}^{\prime}\right) \mathrm{C}=\mathrm{C}=\mathrm{O}, C \equiv$, $\left.-\mathrm{C}_{6} \mathrm{H}_{5}\right]$, and three sets of $\kappa / \kappa^{\prime}$ for the three type of chemically different type of $\mathrm{H}$ atoms $\left[\mathrm{H}_{3} \mathrm{CC}_{5} \mathrm{H}_{4}\right.$, $\mathrm{H}_{3} \mathrm{CC}_{5} \mathrm{H}_{4}$, Co-CO, $\left.\mathrm{R}\left(\mathrm{R}^{\prime}\right) \mathrm{C}=\mathrm{C}=\mathrm{O}, \mathrm{R}\left(\mathrm{R}^{\prime}\right) \mathrm{C}=\mathrm{C}=\mathrm{O}, \mathrm{C} \equiv,-\mathrm{C}_{6} \mathrm{H}_{5}\right]$ were used and refined. For the hydrogen atoms, however, the $\kappa^{\prime}$ parameter was fixed to unity. In addition, the valence populations and dipole parameters for the $\mathrm{H}$ atoms within the methyl, cyclopentadienyl, and phenyl groups were constraint to be the same in each group. In the final cycles of refinement, all parameters were allowed to vary 
[within the limits of the above constraints and restraints] and all data available ( $\mathrm{I}>0)$ were used. No significant extinction was found. The Hirshfeld rigid bond test ${ }^{10}$ on atomic displacement parameters deserves some comments. It is respected for most of the interatomic bonds involving light atoms (C, O, $\mathrm{H})$. As expected, the Mn-C and Co-C bonds exhibit rather high discrepancies, which is usual for atoms with highly different atomic weight. Some of the carbonyl groups also do not fulfil the rigid bond test ; this might be due to an incomplete deconvolution of thermal smearing effects or to an inadequacy of the $\mathrm{C}$ and $\mathrm{O}$ radial functions. According to the results of our refinement, it seems that the radial function can not be better optimised.

$1 \quad$ CrysAlis RED, Oxford Diffraction Ltd., Version 1.171.27p20 beta (release 09.16.2005)

2 Analytical numeric absorption correction using a multifaceted crystal model based on expressions derived by R.C. Clark \& J.S. Reid.

$3 \quad$ Blessing, R. H., J. Appl. Crystallogr. 1989, 22, 396.

4 Altomare, A.; Burla, M. C.; Camalli, G.; Cascarano, G.; Giacovazzo, C.; Gualiardi, A.; Polidori, G. J. Appl. Cryst. 1994, 27, 435.

5 G. M. Sheldrick SHELX97 - Programs for Crystal Structure Analysis (Release 97-2), Institüt für Anorganische Chemie der Universität, Tammanstrasse 4, D-3400 Göttingen, Germany, 1998.

$6 \quad$ Farrugia, L. J. J. Appl. Cryst. 1999, 32, 837-838.

$7 \quad$ Hansen, N. K.; Coppens, P. Acta Crystallogr. 1978, A34, 909.

8 Clementi, E.; Roetti, C. At. Data Nucl. Data Tables 1974, 14, 177.

9 International Tables Vol C - A. J. C. Wilson, Ed., International Tables for Crystallography, Volume C, Kluwer Academic Publishers, Dordrecht, The Netherlands, 1995, Tables 4.2.6.8.

10 Hirsfeld, F. L. Acta Crystallogr. 1976, A32, 239 
Table S1 Crystal data and structure refinement for compound 1.

\begin{tabular}{|c|c|c|}
\hline \multicolumn{3}{|c|}{ Crystal Data } \\
\hline Empirical formula & $\mathrm{C}_{30} \mathrm{H}_{17} \mathrm{Co}_{2} \mathrm{MnO}_{9}$ & \\
\hline Formula weight & 694.24 & \\
\hline Temperature & $100(2) \mathrm{K}$ & \\
\hline Wavelength & $0.71069 \AA$ & \\
\hline Crystal system & triclinic & \\
\hline Space group & $P-1$ & \\
\hline \multirow[t]{3}{*}{ Unit cell dimensions } & $a=9.2994(5) \AA$ & $\alpha=98.870(4)^{\circ}$. \\
\hline & $b=10.1733(5) \AA$ & $\beta=90.080(4)^{\circ}$. \\
\hline & $c=14.6154(7) \AA$ & $\gamma=97.201(4)^{\circ}$. \\
\hline Volume & $1355.08(12) \AA^{3}$ & \\
\hline $\mathrm{Z}$ & 2 & \\
\hline Density (calculated) & $1.702 \mathrm{Mg} / \mathrm{m}^{3}$ & \\
\hline Absorption coefficient & $1.728 \mathrm{~mm}^{-1}$ & \\
\hline $\mathrm{F}(000)$ & 696 & \\
\hline Crystal size & $0.22 \times 0.58 \times 0.66$ & \\
\hline Theta range & 3.2 to $136.8^{\circ}$. & \\
\hline Reflections collected & 119756 & \\
\hline Independent reflections & 35022 & \\
\hline Absorption correction & numerical & \\
\hline$T_{\min } / T_{\max }=$ & $0.346 / 0.735$ & \\
\hline \multicolumn{3}{|c|}{ MOLLY refinement } \\
\hline Theta range & \multicolumn{2}{|l|}{3.2 to $136.8^{\circ}$} \\
\hline Completeness to theta $=102.6^{\circ}$ & \multicolumn{2}{|l|}{$69 \%$} \\
\hline Average redundancy & \multicolumn{2}{|l|}{3.7} \\
\hline Refinement method & \multicolumn{2}{|c|}{ Full-matrix least-squares on $\mathrm{F}$} \\
\hline Data & \multicolumn{2}{|l|}{33984} \\
\hline Goodness-of-fit on F & \multicolumn{2}{|l|}{1.86} \\
\hline $\mathrm{R}$ indices $(\mathrm{I}>0)$ & \multicolumn{2}{|c|}{$\mathrm{R}=0.0430, \mathrm{wR}=0.0222$} \\
\hline R2 indices ( $I>0)$ & \multicolumn{2}{|c|}{$\mathrm{R} 2=0.0312, \mathrm{wR} 2=0.0450$} \\
\hline
\end{tabular}


Table S2 Atomic coordinates for compound $\mathbf{1}$ after the multipolar refinement.

\begin{tabular}{|c|c|c|c|}
\hline & $x / a$ & $\mathrm{y} / \mathrm{b}$ & $z / c$ \\
\hline $\mathrm{C} 1$ & $0.11084(5)$ & $0.30366(4)$ & $0.62726(3)$ \\
\hline C2 & $\odot .06901(4)$ & $0.33005(4)$ & $0.80522(3)$ \\
\hline C3 & $-0.13241(4)$ & $0.39811(4)$ & $0.64987(2)$ \\
\hline C4 & $-0.52652(4)$ & $\odot .23272(4)$ & $0.77081(3)$ \\
\hline C5 & $-0.53127(5)$ & $-0.03711(4)$ & $0.70829(3)$ \\
\hline c6 & $-0.46278(5)$ & $0.16328(5)$ & $0.58622(3)$ \\
\hline C7 & $0.00029(5)$ & $0.02827(4)$ & $0.69616(3)$ \\
\hline $\mathrm{C} 8$ & $-0.16333(5)$ & $\odot .08279(4)$ & $0.54579(3)$ \\
\hline C9 & $-\odot .25 \odot 40(5)$ & $-0.13552(4)$ & $0.64213(3)$ \\
\hline C10 & $-\odot .17080(4)$ & $\odot .35715(3)$ & $0.73745(2)$ \\
\hline C11 & $-0.21947(4)$ & $\odot .21383(3)$ & $0.73508(2)$ \\
\hline $\mathrm{C} 12$ & $-\odot .25670(4)$ & $0.11345(3)$ & $0.78500(2)$ \\
\hline $\mathrm{C} 21$ & $0.24283(4)$ & $0.56327(4)$ & $0.67501(3)$ \\
\hline $\mathrm{C} 22$ & $0.11121(5)$ & $0.60403(4)$ & $0.64575(3)$ \\
\hline H22 & $0.083(1)$ & $0.605(1)$ & $0.5740(2)$ \\
\hline $\mathrm{C} 23$ & $\odot .02822(5)$ & $\odot .63965(4)$ & $0.72585(4)$ \\
\hline H23 & $-0.0782(5)$ & $\odot .6723(9)$ & $0.7253(7)$ \\
\hline C24 & $0.10620(6)$ & $0.61869(5)$ & $\odot .80490(3)$ \\
\hline H24 & $0.071(1)$ & $0.634(1)$ & $0.8760(2)$ \\
\hline C25 & $\odot .23875(5)$ & $\odot .57081(5)$ & $0.77288(3)$ \\
\hline H25 & $0.3213(7)$ & $0.5415(9)$ & $0.8151(5)$ \\
\hline C26 & $0.36465(5)$ & $0.52536(5)$ & $0.61355(4)$ \\
\hline H26 & $0.4413(8)$ & $\odot .6159(5)$ & $0.6130(6)$ \\
\hline H27 & $0.3251(9)$ & $0.4833(9)$ & $0.5434(3)$ \\
\hline H28 & $0.4201(9)$ & $0.4502(6)$ & $0.6405(6)$ \\
\hline C31 & $-0.23874(4)$ & $0.45223(4)$ & $0.80775(2)$ \\
\hline C32 & $-0.31947(4)$ & $\odot .54615(4)$ & $0.77886(3)$ \\
\hline H32 & $-0.3326(9)$ & $0.5491(8)$ & $0.7056(2)$ \\
\hline C33 & $-\odot .38498(5)$ & $\odot .63455(5)$ & $0.84354(4)$ \\
\hline H33 & $-0.4410(8)$ & $0.7091(6)$ & $0.8180(5)$ \\
\hline C34 & $-0.37343(6)$ & $\odot .62770(5)$ & $0.93772(4)$ \\
\hline H34 & $-0.4231(9)$ & $0.6970(7)$ & $0.9879(4)$ \\
\hline C35 & $-\odot .29436(6)$ & $\odot .53309(5)$ & $0.96701(3)$ \\
\hline H35 & $-0.278(1)$ & $0.5308(9)$ & $1.0401(2)$ \\
\hline C36 & $-\odot .22623(5)$ & $\odot .44669(5)$ & $0.90266(3))$ \\
\hline H36 & $-0.1621(8)$ & $0.3743(6)$ & $0.9238(5)$ \\
\hline C41 & $-0.25479(4)$ & $0.07387(4)$ & $0.87737(2)$ \\
\hline C42 & $-0.17762(5)$ & $-\odot .02919(5)$ & $0.89541(3)$ \\
\hline H42 & $-0.1198(8)$ & $-0.0813(8)$ & $0.8397(4)$ \\
\hline C43 & $-0.17515(6)$ & $-\odot .0650 \odot(6)$ & $0.98385(3)$ \\
\hline H43 & $-0.1157(9)$ & $-0.1463(6)$ & $\odot .9950(6)$ \\
\hline C44 & $-0.25108(6)$ & $\odot .00 \odot 40(6)$ & $1.05542(3)$ \\
\hline H44 & $-0.249(1)$ & $-0.0289(8)$ & $1.1236(3)$ \\
\hline C45 & $-0.32976(7)$ & $0.10199(6)$ & $1.03784(3)$ \\
\hline H45 & $-0.3913(8)$ & $0.1552(8)$ & $1.0910(4)$ \\
\hline C46 & $-0.33223(6)$ & $0.13814(5)$ & $0.94972(3)$ \\
\hline H46 & $-\odot .3968(8)$ & $0.2153(6)$ & $0.9373(6)$ \\
\hline 01 & $0.15437(5)$ & $0.22795(4)$ & $0.56987(3)$ \\
\hline 02 & $0.07488(4)$ & $0.26769(4)$ & $0.86400(2)$ \\
\hline 03 & $-0.18737(4)$ & $0.41225(4)$ & $0.57809(2)$ \\
\hline 04 & $-0.59610(4)$ & $0.30018(4)$ & $0.81677(3)$ \\
\hline 05 & $-0.59692(4)$ & $-0.13637(4)$ & $0.71754(3)$ \\
\hline 06 & $-0.48978(5)$ & $0.18586(5)$ & $0.51433(3)$ \\
\hline 07 & $0.11529(4)$ & $0.01805(4)$ & $0.71924(3)$ \\
\hline 08 & $-\odot .15457(5)$ & $0.10977(4)$ & $0.47301(2)$ \\
\hline 09 & $-0.29297(4)$ & $-0.24723(3)$ & $0.62883(3)$ \\
\hline Mn1 & $0.056471(1)$ & $0.431449(1)$ & $0.714151(1)$ \\
\hline Co1 & $-0.420233(1)$ & $0.122307(1)$ & $0.699924(1)$ \\
\hline $\mathrm{Co} 2$ & $-0.183637(1)$ & $0.042575(1)$ & $0.663566(1)$ \\
\hline
\end{tabular}


Table S3 Anisotropic or isotropic displacement parameters for compound $\mathbf{1}$ after the multipolar refinement. The anisotropic displacement factor exponent takes the form: $-2 \pi^{2}\left[h^{2} a^{* 2} U^{11}+\ldots+2 h k\right.$ a* $\left.\mathrm{b}^{*} \mathrm{U}^{11}\right]$.

\begin{tabular}{|c|c|c|c|c|c|c|}
\hline & U11 & U22 & U33 & U12 & U13 & U23 \\
\hline $\mathrm{C} 1$ & $0.0177(2)$ & $0.0147(1)$ & $0.0184(2)$ & $0.0003(1)$ & $0.0072(1)$ & $0.0006(1)$ \\
\hline $\mathrm{C} 2$ & $0.0132(2)$ & $0.0145(2)$ & $0.0151(2)$ & $0.0005(1)$ & $-0.0012(1)$ & $0.0050(1)$ \\
\hline C3 & $0.0148(1)$ & $0.0122(1)$ & $0.0086(1)$ & $0.0003(1)$ & $-0.00023(9)$ & $0.00281(9)$ \\
\hline C4 & $0.0124(1)$ & $0.0157(1)$ & $0.0183(1)$ & $0.0038(1)$ & $0.0018(1)$ & $0.0015(1)$ \\
\hline C5 & $0.0146(1)$ & $0.0129(1)$ & $0.0195(2)$ & $-0.0013(1)$ & $0.0013(1)$ & $0.0025(1)$ \\
\hline C6 & $0.0179(2)$ & $0.0209(2)$ & $0.0131(1)$ & $0.0028(1)$ & $-0.0040(1)$ & $0.0043(1)$ \\
\hline C7 & $0.0132(1)$ & $0.0177(1)$ & $0.0149(1)$ & $0.0047(1)$ & $0.0005(1)$ & $0.0005(1)$ \\
\hline $\mathrm{C} 8$ & $0.0235(2)$ & $0.0158(1)$ & $0.0100(1)$ & $0.0043(1)$ & $0.0033(1)$ & $0.0018(1)$ \\
\hline C9 & $0.0174(1)$ & $0.0103(1)$ & $0.0153(1)$ & $0.0014(1)$ & $-0.0010(1)$ & $0.0020(1)$ \\
\hline $\mathrm{C} 10$ & $0.0118(1)$ & $0.0093(1)$ & $0.0085(1)$ & $0.00095(9)$ & $0.00075(9)$ & $0.00163(8)$ \\
\hline $\mathrm{C} 11$ & $0.0110(1)$ & $0.0089(1)$ & $0.0091(1)$ & $0.00080(9)$ & $0.00056(9)$ & $0.00169(8)$ \\
\hline $\mathrm{C} 12$ & $0.0117(1)$ & $0.0104(1)$ & $0.0084(1)$ & $0.00133(9)$ & $0.00052(9)$ & $0.00268(9)$ \\
\hline $\mathrm{C} 21$ & $0.0132(1)$ & $0.0160(1)$ & $0.0170(1)$ & $-0.0020(1)$ & $0.0007(1)$ & $0.0056(1)$ \\
\hline $\mathrm{C} 22$ & $0.0162(1)$ & $0.0162(1)$ & $0.0198(2)$ & $-0.0007(1)$ & $-0.0001(1)$ & $0.0087(1)$ \\
\hline C23 & $0.0177(2)$ & $0.0124(1)$ & $0.0280(2)$ & $0.0012(1)$ & $0.0036(1)$ & $0.0052(1)$ \\
\hline C24 & $0.0240(2)$ & $0.0153(1)$ & $0.0185(2)$ & $-0.0029(1)$ & $0.0037(1)$ & $-0.0010(1)$ \\
\hline $\mathrm{C} 25$ & $0.0169(2)$ & $0.0179(2)$ & $0.0170(1)$ & $-0.0040(1)$ & $-0.0026(1)$ & $0.0036(1)$ \\
\hline C26 & $0.0155(2)$ & $0.0240(2)$ & $0.0242(2)$ & $-0.0014(1)$ & $0.0048(1)$ & $0.0065(2)$ \\
\hline C31 & $0.0122(1)$ & $0.0103(1)$ & $0.0105(1)$ & $0.00133(9)$ & $0.00132(9)$ & $0.00045(9)$ \\
\hline C32 & $0.0145(1)$ & $0.0126(1)$ & $0.0194(2)$ & $0.0037(1)$ & $0.0024(1)$ & $0.0032(1)$ \\
\hline C33 & $0.0185(2)$ & $0.0148(2)$ & $0.0316(2)$ & 0.0055 (1) & $0.0074(2)$ & $0.0011(1)$ \\
\hline C34 & $0.0243(2)$ & $0.0198(2)$ & $0.0280(2)$ & $0.0034(2)$ & $0.0109(2)$ & $-0.0060(2)$ \\
\hline C35 & $0.0265(2)$ & $0.0259(2)$ & $0.0145(2)$ & $0.0029(2)$ & $0.0060(1)$ & $-0.0047(1)$ \\
\hline C36 & $0.0199(2)$ & $0.0198(2)$ & $0.0101(1)$ & $0.0036(1)$ & $0.0016(1)$ & $-0.0002(1)$ \\
\hline $\mathrm{C} 41$ & $0.0140(1)$ & $0.0121(1)$ & $0.0094(1)$ & $0.00072(10)$ & $-0.00015(9)$ & $0.00361(9)$ \\
\hline C42 & $0.0245(2)$ & $0.0194(2)$ & $0.0157(1)$ & $0.0071(1)$ & $0.00013(13)$ & $0.0084(1)$ \\
\hline C43 & $0.0286(2)$ & $0.0266(2)$ & $0.0198(2)$ & $0.0037(2)$ & $-0.0039(2)$ & $0.0139(2)$ \\
\hline C44 & $0.0305(2)$ & $0.0306(2)$ & $0.0135(1)$ & $-0.0023(2)$ & $-0.0026(1)$ & $0.0118(1)$ \\
\hline C45 & $0.0414(3)$ & $0.0360(3)$ & $0.0125(2)$ & $0.0098(2)$ & $0.0079(2)$ & $0.0092(2)$ \\
\hline C4 6 & $0.0312(2)$ & $0.0269(2)$ & $0.0125(1)$ & $0.0116(2)$ & $0.0076(1)$ & $0.0076(1)$ \\
\hline 01 & $0.0305(2)$ & $0.0211(2)$ & $0.0309(2)$ & $0.0004(1)$ & $0.0180(1)$ & $-0.0054(1)$ \\
\hline $\mathrm{O} 2$ & $0.0212(2)$ & $0.0225(2)$ & $0.0191(2)$ & $0.0010(1)$ & $-0.0039(1)$ & $0.0112(1)$ \\
\hline 03 & $0.0221(1)$ & $0.0245(1)$ & $0.0109(1)$ & $0.0004(1)$ & $-0.00325(10)$ & $0.00699(10)$ \\
\hline 04 & $0.0189(1)$ & $0.0241(2)$ & $0.0305(2)$ & $0.0091(1)$ & $0.0071(1)$ & $-0.0017(1)$ \\
\hline 05 & $0.0257(2)$ & $0.0154(1)$ & $0.0386(2)$ & $-0.0064(1)$ & $0.0061(1)$ & $0.0055(1)$ \\
\hline 06 & $0.0337(2)$ & $0.0406(2)$ & $0.0162(1)$ & $0.0062(2)$ & $-0.0080(1)$ & $0.0106(1)$ \\
\hline 07 & $0.0149(1)$ & $0.0330(2)$ & $0.0234(1)$ & $0.0093(1)$ & $-0.0021(1)$ & $0.0002(1)$ \\
\hline 08 & $0.0473(2)$ & $0.0294(2)$ & $0.0108(1)$ & $0.0091(2)$ & $0.0073(1)$ & $0.0061(1)$ \\
\hline 09 & $0.0292(2)$ & $0.0105(1)$ & $0.0279(2)$ & $-0.0015(1)$ & $-0.0038(1)$ & $0.0041(1)$ \\
\hline Mn1 & $0.01052(2)$ & $0.00993(2)$ & $0.01098(2)$ & $-0.00031(1)$ & $0.00101(1)$ & $0.00265(1)$ \\
\hline $\mathrm{Col}$ & $0.00937(2)$ & $0.00993(2)$ & $0.01005(2)$ & $0.00067(1)$ & $-0.00072(1)$ & $0.00177(1)$ \\
\hline $\mathrm{Co} 2$ & $0.01139(2)$ & $0.00889(2)$ & $0.00872(2)$ & $0.00188(1)$ & $0.00078(1)$ & $0.00076(1)$ \\
\hline
\end{tabular}


Table S4. Selected bond lengths $[\AA]$ and angles $\left[{ }^{\circ}\right]$ for compound 1 after the multipolar refinement

$\begin{array}{ll}\text { C01-C4 } & 1.7984(4) \\ \text { C01-C5 } & 1.8311(4) \\ \text { C01-C6 } & 1.8290(5) \\ \text { C01-C11 } & 2.0076(4) \\ \text { C01-C12 } & 1.9826(4) \\ \text { C02-C7 } & 1.8042(5) \\ \text { C02-C8 } & 1.8343(4) \\ \text { C02-C9 } & 1.8190(4) \\ \text { C02-C11 } & 1.9555(4) \\ \text { C02-C12 } & 1.9655(3) \\ \text { Mn1-C1 } & 1.7933(4) \\ \text { Mn1-C2 } & 1.8173(4) \\ \text { Mn1-C3 } & 1.9572(4) \\ \text { Mn1-C10 } & 2.1989(4) \\ \text { 01-C1 } & 1.1578(6) \\ \text { 02-C2 } & 1.1490(5) \\ \text { 03-C3 } & 1.2025(5) \\ \text { 04-C4 } & 1.1451(6) \\ \text { 05-C5 } & 1.1395(5) \\ \text { 06-C6 } & 1.1437(6) \\ \text { 07-C7 } & 1.1428(6) \\ \text { 08-C8 } & 1.1394(5) \\ \text { 09-C9 } & 1.1423(5) \\ \text { C3-C10 } & 1.4381(4) \\ \text { C10-C11 } & 1.4666(4) \\ \text { C10-C31 } & 1.4963(5) \\ \text { C11-C12 } & 1.3553(5) \\ \text { C01-C02 } & 2.4696(1)\end{array}$

Mn1-C1- 01

Mn1-C2- 02

Mn1-C3-03

Mn1-C3-C10

$03-\mathrm{C} 3-\mathrm{C} 10$

C01-C4-04

C01-C5-05

C01-C6-06

$\mathrm{Co} 2-\mathrm{C} 7-07$

C02-C8-08

C02-C9-09

Mn1-C10-C3

Mn1-C10-C11

Mn1-C10-C31

C3-C10-C11

C3-C10-C31

C11-C10-C31

C01-C11-Co2

C01-C11-C10

C01-C11-C12

Co2-C11-C10

Co2-C11-C12

C10-C11-C12

Co1-C12-Co2

C01-C12-C11

Co1-C12-C41

Co2-C12-C11

Co2 - C12-C41

C11-C12-C41
$174.97(4)$

178.44(4)

$140.16(3)$

$79.11(2)$

140.59(4)

$178.21(4)$

$176.62(4)$

178.44(5)

$177.94(4)$

$177.82(4)$

$179.67(4)$

60.94(2)

121.61(3)

$111.82(3)$

$115.93(3)$

118.44(3)

$116.63(3)$

$77.08(1)$

$125.40(3)$

$69.15(2)$

$138.35(2)$

$70.18(2)$

$146.54(3)$

$77.44(1)$

$71.14(2)$

$131.07(3)$

$69.38(2)$

$134.42(3)$

145.19 (3)

$\begin{array}{ll}\text { C4-C01-C5 } & 98.56(2) \\ \text { C4-C01-C6 } & 99.37(2) \\ \text { C4-C01-C11 } & 100.44(2) \\ \text { C4-C01-C12 } & 102.03(2) \\ \text { C5-C01-C6 } & 106.00(2) \\ \text { C5-C01-C11 } & 138.74(2) \\ \text { C5-C01-C12 } & 100.48(2) \\ \text { C6-C01-C11 } & 106.55(2) \\ \text { C6-C01-C12 } & 142.80(2) \\ \text { C11-C01-C12 } & 39.71(1) \\ \text { C7-C02-C8 } & 102.31(2) \\ \text { C7-Co2-C9 } & 97.76(2) \\ \text { C7-Co2-C11 } & 103.31(2) \\ \text { C7-Co2-C12 } & 99.83(2) \\ \text { C8-C02-C9 } & 102.23(2) \\ \text { C8-C02-C11 } & 102.53(2) \\ \text { C8-Co2-C12 } & 140.78(2) \\ \text { C9-Co2-C11 } & 142.99(2) \\ \text { C9-C02-C12 } & 106.36(2) \\ \text { C11-Co2-C12 } & 40.44(1) \\ \text { C1-Mn1-C2 } & 92.49(2) \\ \text { C1-Mn1-C3 } & 86.34(2) \\ \text { C1-Mn1-C10 } & 103.17(2) \\ \text { C2-Mn1-C3 } & 113.25(2) \\ \text { C2-Mn1-C10 } & 76.43(1) \\ \text { C3-Mn1-C10 } & 39.96(1) \\ \text { C10-Mn1-C22 } & 120.96(2) \\ \text { C10-Mn1-C23 } & 96.59(2)\end{array}$


Table S5. Multipolar refinement parameters for compound 1.

atom: $\quad \mathrm{C} 1$

\begin{tabular}{|c|c|c|c|c|c|c|c|}
\hline \multirow{2}{*}{\multicolumn{3}{|c|}{$\begin{array}{l}\text { Coordinate system } 01 \\
\text { Kappa1 kappa2 1.020638 }\end{array}$}} & \multirow{2}{*}{\multicolumn{5}{|c|}{$\begin{array}{l}Z \quad{ }_{0}^{C 2} \\
0.867175\end{array}$}} \\
\hline & & & & & & & \\
\hline Pv, $\mathrm{P} \quad 00^{\circ}$ & 4.159 & 0 . & & & & & \\
\hline Dipoles & & 0 . & -.083 & & & & \\
\hline quadrupoles & 0.461 & 0 . & 0 . & 0 . & 0 . & & \\
\hline octupoles & 0.098 & 0 . & 0 . & 0 . & 0. & 0 . & 0 . \\
\hline \multicolumn{8}{|l|}{ atom: $\quad$ C2 } \\
\hline coordinate syst & tem & $\mathrm{O} 2$ & & $\mathrm{C} 1$ & $\mathrm{Y}$ & & \\
\hline Kappal, kappa2 & 1.02063 & & 0.86717 & & & & \\
\hline $\mathrm{PV}, \mathrm{P} 00$ & 4.119 & 0 . & & & & & \\
\hline dipoles & 0.003 & -.029 & -.028 & & & & \\
\hline quadrupoles & 0.465 & -.022 & 0.009 & -.019 & 0.051 & & \\
\hline octupoles & 0.159 & -.027 & 0.014 & 0.024 & -.021 & -.011 & -.046 \\
\hline
\end{tabular}

\section{atom: C3}

\begin{tabular}{llll} 
Coordinate system & Mn1 & \multicolumn{1}{c}{ C10 } & Y \\
Kappa1, kappa2 1.001945 & 0.759450 &
\end{tabular}

$\begin{array}{lll}\text { Kappa1, kappa2 } & 1.001945 \\ \text { Pv, P00 } & 4.1410 .\end{array}$

Dipoles

quadrupoles

$\begin{array}{rrr}-.100 & -.046 & -.064 \\ -.382 & 0.029 & 0.009\end{array}$

$\begin{array}{lllll}.382 & 0.029 & 0.009 & -.067 & 0.329\end{array}$

atom: $\quad \mathrm{C4}$

coordinate system 04

Kappa1, kappa2 1.040998

Pv, P0O 3.9530.

dipoles

$\begin{array}{ll}3.953 & 0 . \\ 0 . & 0 .\end{array}$

$\begin{array}{lll}\text { quadrupoles } & 0.469 & 0 . \\ \text { octupoles } & 0.158 & 0 .\end{array}$

atom: C5

Coordinate system 05

Kappa1, kappa2 1.040998

Pv, P00
dipoles

dipoles 0.0.

$\begin{array}{lll}\text { quadrupoles } & 0.446 & 0 . \\ \text { octupoles } & 0.111 & 0 .\end{array}$

atom: $\quad \mathrm{C} 6$

coordinate system 06

Kappa1, kappa2 1.040998

Pv, P00 4.0560.

dipoles

0.00.

quadrupoles

$\begin{array}{ll}0.404 & 0 . \\ 0.106 & 0 .\end{array}$

$\begin{array}{lllll}Z & C 5 & Y & & \\ 0.932602 & & & \\ 0.020 & & & & \\ 0 . & 0 . & 0 . & & \\ 0 . & 0 . & 0 . & 0 . & 0 . \\ & & & & \\ Z & C 6 & Y & & \\ 0.932602 & & & \\ 0.013 & & & & \\ 0 . & 0 . & 0 . & & \\ 0 . & 0 . & 0 . & 0 . & 0 . \\ Z & & & & \\ Z & C 4 & Y & & \\ 0.932602 & & & \\ -.009 & & & & \\ 0 . & 0 . & 0 . & 0 . & 0 . \\ 0 . & 0 . & 0 . & \end{array}$

atom: $\quad$ C7

$\begin{array}{lllll}\text { Coordinate system } & 07 & Z & \text { C }\end{array}$

Pv, P0O 4.1430.

0.932602

$\begin{array}{ll}4.143 & 0 . \\ 0.435 & 0 . \\ 0.435 & 0 .\end{array}$

0.018

quadrupoles $\quad 0.435$

0.

tom: $\quad C 8$

$\begin{array}{lll}\text { Kappa1, kappa2 } & 1.040998 \\ \text { Pv, P00 } & 4.0640 .\end{array}$

dipoles

$\begin{array}{llll}\text { dipoles } & 0.064 & 0 . & 0.007 \\ \text { quadrupoles } & 0.434 & 0 . & 0 .\end{array}$

$\mathrm{Z}$
$0.932602^{C 9} \quad \mathrm{Y}$

atom: $\quad$ C9

coordinate system

(a)

Pv, P0O

dipoles $\quad 0.9720$.

$\begin{array}{lll}\text { quadrupoles } & 0.430 & 0 \\ \text { octupoles } & 0.089 & 0\end{array}$

0.0 .00$.

0.031

atom: $\quad \mathrm{C} 10$

coordinate system Mn1 X C3 $\quad$ Y

Kv

$\begin{array}{llllll}\text { dipoles } & -.006 & -.042 & 0.001 & & \\ \text { quadrupoles } & 0.035 & -.001 & -.013 & -.041 & 0.017\end{array}$

$\begin{array}{llllllll}\text { quadrupoles } & 0.035 & -.001 & -.013 & -.041 & 0.017 & & \\ \text { octupoles } & -.006 & -.205 & -.119 & -.023 & 0.014 & -.054 & 0.098\end{array}$

atom: $\quad$ C11

coordinate system C10 X C12 Y

Kappa1, kappa2 1.022906 0.824224

Pv, P0O

dipoles $\quad-.073 \quad 0.028 \quad-.015$

$\begin{array}{llllllll}\text { quadrupoles } & -.195 & -.032 & -.008 & 0.180 & -.079 & & \\ \text { octupoles } & -.055 & -.081 & -.083 & -.003 & 0.021 & 0.218 & -.128\end{array}$

atom: $\quad \mathrm{C} 12$

coordinate system C11 X C41 Y

Kappa1, kappa2 $1.022906 \quad 0.824224$

dipoles $\quad 0.036 \quad 0.012 \quad 0.005$

$\begin{array}{llllll} & -.036 & 0.012 & 0.005 & & \\ \text { quatrupoles } & -.005 & 0.029 & -.005 & 0.105 & -.071\end{array}$

atom: $\quad$ C21

$\begin{array}{lllll}\text { Coordinate system CENT1 } & \text { X } & \text { Mn1 } & \text { Z } \\ \text { Kappa1, kappa2 } 0.993578 & 0.856280 & \end{array}$

dipoles $\quad 0.206 \quad 0.158 \quad-.027$

$\begin{array}{lllll}-.299 & -.028 & 0.052 & 0.014 & 0.067\end{array}$

$\begin{array}{llllllll}\text { quadrupoles } & -.299 & -.028 & 0.052 & 0.014 & 0.067 & & \\ \text { octupoles } & 0.046 & 0.005 & -.040 & 0.021 & -.013 & -.399 & 0.004\end{array}$ 
atom: $\quad$ C22

CENT1 X Mn1 Z

Pv P00

dipoles

4.192
0.082

0.087
-.002

.001

quadrupoles

$\begin{array}{lllll}-.298 & 0.002 & 0.048 & 0.085 & -.020 \\ 0.025 & 0.030 & -.017 & -.006 & 0.015\end{array}$

$-.389 \quad 0.091$

atom: $\mathrm{H} 22$

$\begin{array}{llll}\text { Coordinate system } & \text { C22 } \quad \text { X } & \text { C23 } & \text { Y } \\ \text { Kappa1, kappa2 } & 1.150068 & 1.200000^{-2}\end{array}$

dipoles

$0.721 \quad 0$.

atom: $\quad$ C23

CENT1 X Mn1 Z

Pv, P00 4.126

Dipoles -.049 $-.067 \quad 0.042$

$\begin{array}{llllll}\text { quadrupoles } & -.246 & 0.079 & -.035 & 0.038 & -.059\end{array}$

$\begin{array}{lllllllll}\text { octupoles } & 0 . & 0.072 & 0.018 & -.001 & -.036 & -.355 & -.023\end{array}$

atom: $\mathrm{H} 23$

$\begin{array}{llll}\text { Coordinate system C23 } & \text { X } & \text { C24 } & \text { Y } \\ \text { Kappa1, kappa2 } 1.150068 & 1.200000 & \end{array}$ 0.7210.

atom: C24

CENT1 X $\quad$ Mn1 Z

Kappa1, kappa2 0.993578

dipoles 4.1190

quadrupoles

$0.057 \quad 0.068$

octupoles

$\begin{array}{lllll}0.035 & -.001 & -.085 & 0.051 & 0.040 \\ 0.093 & -.027 & -.021 & 0.010\end{array}$

$-.369 \quad 0.057$

\section{atom: $\mathrm{H} 24$}

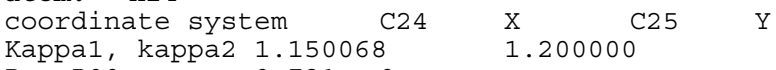

Ka, PO

0.7210

atom: C25

CENT1 X $\quad$ Mn1 Z

Kv, POO Kapa2 0.993578

dipoles

$\begin{array}{ll}3.949 & 0 . \\ -.015 & 0.158\end{array}$

quadrupoles

$\begin{array}{lll}-.015 & 0.158 & 0.046 \\ -.168 & -.026 & -.006\end{array}$

octupoles

$\begin{array}{lllll}.083 & 0.054 & -.0067 & 0.093 & 0.041\end{array}$

$-.314 \quad 0.012$

\section{atom: $\mathrm{H} 25$}

$\begin{array}{lll}\text { Coordinate system C25 } & \text { X C21 } \\ \text { Kappa1, kappa2 1.150068 } & 1.200000\end{array}$

dipoles

0.7210.

\section{atom: $\quad$ C26}

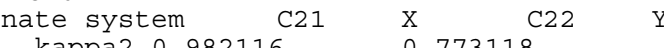

Kappa1, kappa2 0.982116

dipoles

3.9280.

$\begin{array}{llll} & 0.007 & 0.008 & 0.064\end{array}$

$\begin{array}{lllllll} & -.053 & -.040 & -.029 & -.026 & -.036\end{array}$

\section{atom: $\mathrm{H} 26$}

$\begin{array}{lllllll}-.203 & -.225 & 0.083 & -. .140 & 0.034 & 0.280 & -.044\end{array}$

$\begin{array}{llll}\text { Coordinate system C26 } & \text { X C21 } & \text { Y } \\ \text { Kappa1, kappa2 } 1.076790 & 1.200000\end{array}$

.2550 .0

atom: $\quad$ H27

$\begin{array}{lllll}\text { coordinate system C26 } & X & \text { C21 } & \text { Y }\end{array}$

Kappa1, kappa2 1.076790

dipoles

0.2550.

coordinate system C26 X $\mathrm{X}$ C21 $\quad$ Y

Kappa1, kappa2 $1.076790 \quad 1.200000$

$\begin{array}{llll}\text { Pv, P00 } & 0.933 & 0 . & \\ \text { dipoles } & 0.255 & 0 . & 0 .\end{array}$

atom: $\quad$ C31

$\begin{array}{llllll}\text { coordinate system } & \text { C34 } & X & \text { C32 } & Y\end{array}$

Kappa1, kappa2 $1.021513 \quad 0.876546$

0.0150 .

quadrupoles $\begin{array}{llllll}0.015 & -.225 & 0 . & 0 . & -.016 & 0 .\end{array}$

coordinate system C35 X C33 Y

Kappa1, kappa2 1.021513 0.876546

$\begin{array}{llll}\text { Pv, P00 } & 3.942 & 0 . & \\ \text { dipoles } & 0.059 & -.041 & 0 .\end{array}$

$\begin{array}{llll}0.059 & -.041 & 0 . \\ \text { quadrupoles } & -.231 & 0.004 & 0 .\end{array}$

$\begin{array}{llllllllllllll}\text { coordinate system } & \text { C32 } & X & \text { C31 } & \text { Y }\end{array}$

Kappa1, kappa2 1.093340

Pv, P00 $\quad 0.9420$.

\section{atom: $\quad$ C33}

coordinate system C36 X $\quad$ C34 $\quad$ Y

Kappa1, kappa2 $1.021513 \quad 0.876546$

$\begin{array}{llll}\text { Pv, P00 } & 3.878 & 0 . & \\ \text { dipoles } & 0.126 & -.030 & 0 .\end{array}$

$\begin{array}{lllll}0.228 & 0 . & 0 . & -.035 & -.022\end{array}$ 
atom: $\quad$ H33

$\begin{array}{lllll} & & & & \\ \text { C33 } & X & \end{array}$

Kappa1, kappa2 1.093340

dipoles

0.2440.

coordinate system C31 $\quad X \quad$ C35

$.021513 \quad 0.876546$

Pv, P00 $\quad 3.8570$

dipoles

quadrupole
octupoles

$\begin{array}{lll}0.090 & 0 . & 0 \\ -.236 & 0.0 & 0 \\ 0.2 & 0.041 & 0\end{array}$

atom: $\mathrm{H} 34$

$\begin{array}{llll} & & & \\ \text { C34 } & \mathrm{X} & 32 & \end{array}$

dipoles

0.9420

0 .

atom: $\quad$ C35

\begin{tabular}{llll} 
Coordinate system C32 & X & \multicolumn{1}{c}{ C34 } & Y \\
Kappa1, kappa2 1.021513 & 0.876546 &
\end{tabular}

Pv, P00 3.7760.

dipoles

$0.087 \quad 0.025 \quad 0$

quadrupole

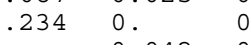

octupoles

0.0 .042

0.001

$-.070$

.01

$0 . \quad-.284-.063$

atom: H35

$\begin{array}{lllll}\text { Coordinate system C35 } & X & \text { C34 } & \text { Y }\end{array}$

Kappa1, kappa2 1.093340

$$
0.2440 .
$$

0 .

tom: $\quad$ C36

atom: C36 $\quad$ C33

dipoles

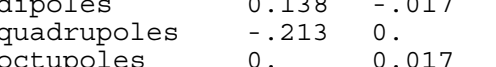

atom: $\mathrm{H3} 6$

coordinate system C36 $\mathrm{X} \quad 36 \quad$ C35

Kappa1, kappa2 1.093340

0.9420.

0 .

atom: $\quad$ C41

$\begin{array}{lllll}\text { C44 } & \text { X } 42 & \text { Y }\end{array}$

Pv, P00 $\quad 3.967 \quad 0$

dipoles $\quad .080 \quad 0$

quadrupoles

$-.2450$.

0

octupoles

$-.069 \quad 0$.

$-.3110$. atom: $\quad$ C42

$\begin{array}{lllll}\text { Coordinate system C45 } & \text { X } & \text { C43 } & \text { Y } \\ \text { Kappa1, kappa2 1.021513 } & 0.876546 & \end{array}$

Kappa1, kappa2 1.0215130.

dipoles

$\begin{array}{lll}0.111 & 0.016 & 0\end{array}$

$\begin{array}{llllll}\text { quadrupoles } & -.227 & 0 . & 0 . & 0.010 & -.013 \\ \text { octupoles } & 0 . & 0.089 & -.031 & 0 . & 0 .\end{array}$

octupoles

$-.3380 .009$

$\begin{array}{llll}\text { Coordinate system C42 } & \text { X } & \text { C41 } & \text { Y }\end{array}$

Kappa1, kappa2 $1.093340 \quad 1.200000$ 0.9420 .00

0 .

atom: $\quad$ C43

$\begin{array}{lllll}\text { te system C46 } & X & \text { C44 } & \text { Y }\end{array}$

Pv, P00 3.7900.

$\begin{array}{llllll}\text { dipoles } & 0.124 & 0.016 & 0 . & & \\ \text { quadrupoles } & -.190 & 0 . & 0 . & 0.016 & -.002\end{array}$

octupoles

$-.190$

$\begin{array}{llll}0.044 & 0.036 & 0.016 & -.002\end{array}$

$-.312-.043$

\section{atom: $\mathrm{H} 43$}

$\begin{array}{llll}\text { Coordinate system C43 } & \text { X } & \text { C42 } & \text { Y } \\ \text { Kappa1, kappa2 } 1.093340 & 1.200000 & \end{array}$

$\begin{array}{llll}\text { Pv, Poo } & 0.942 & 0 . & \\ \text { dipoles } & 0.244 & 0 . & 0\end{array}$

\section{atom: $\quad$ C44}

$\begin{array}{llllllll} & \mathrm{C} 41 & X & \mathrm{C} 45 & Y\end{array}$

Kappal, kappa2 $1.021513 \quad 0.876546$

$\begin{array}{llll}\text { PVipoles } & 3.898 & 0 \\ \text { dipar } & 0.083 & 0 .\end{array}$

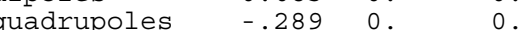

$\begin{array}{lllllllll}\text { octupoles } & 0 . & 0.042 & 0 . & 0 . & 0 . & -.351 & 0 .\end{array}$

atom: $\quad$ H44

$\begin{array}{lllll}\text { Coordinate system C44 } & \text { X } 043 & \text { Y } \\ \text { Kappa1, kappa2 } 1.093340 & 1.200000 & \end{array}$

$\begin{array}{llll}\text { Pv, Poo } & 0.942 & 0 . & \\ \text { dipoles } & 0.244 & 0 . & 0\end{array}$

atom: $\quad \mathrm{C} 45$

$\begin{array}{lllll}\mathrm{C} 42 & \mathrm{X} & \mathrm{C} 44\end{array}$

$\begin{array}{llll}\text { dipoles } & 0.123 & 0.014 & 0 .\end{array}$

quadrupoles $\begin{array}{llllll}0.124 & 0 . & 0 . & -.026 & -.026\end{array}$

$\begin{array}{lllllllll}\text { octupoles } & 0 . & -.023 & -.046 & 0 . & 0 . & -.301 & -.060\end{array}$

\section{atom: 445}

$\begin{array}{llll}\text { Coordinate system C45 } & \text { X } & \text { C44 } & \text { Y } \\ \text { Kappa1, kappa2 } 1.093340 & 1.200000 & \end{array}$

Kappa1, kappa2 $1.093340 \quad 1.200000$

$\begin{array}{llll}\text { Pv, P00 } & 0.942 & 0 . & \\ \text { dipoles } & 0.244 & 0 . & 0 .\end{array}$ 
atom: $\quad$ C46

$\begin{array}{lllll} & & & & \end{array}$

$\mathrm{PV}, \mathrm{P} 003.884$. 3.0

dipoles $0.111-.0100$

$\begin{array}{llllll}\text { quadrupoles } & -.144 & 0 . & 0 . & 0.007 & 0.007\end{array}$

octupoles

$\begin{array}{llll}\text { Coordinate system C46 } & \text { X } & \text { C45 } & \text { Y } \\ \text { Kappa1, kappa2 } 1.093340 & 1.200000 & \end{array}$

dipoles 0.244 . 0.

atom: 01

$\begin{array}{lllllllllllllll}\text { coordinate system } & \text { Mn1 } & \text { Z } & \text { C2 } & \text { Y }\end{array}$

kappa2 $0.999610 \quad 1.140941$

PV, P00 6.2050.

$\begin{array}{lll}\text { dipoles } 0 . & 0 . & 0.071 \\ \text { quadrupoles } & 0.124 & 0 .\end{array}$

octupoles

0.014

\section{atom: 02}

$\begin{array}{lllll}\text { Coordinate system } & \text { Mn1 } & \text { Z } & \text { C1 } & \text { Y } \\ \text { Kappa1, kappa2 } 0.999610 & 1.140941 & \end{array}$

dipoles $0.001-.021-.034$

$\begin{array}{llllllll} & 0.122 & -.004 & -.006 & -.029 & 0.002\end{array}$

$\begin{array}{llllll}-.007 & -.002 & -.017 & 0.011 & 0.003 & -.010\end{array}$

coordinate system $\quad$ C3 $\quad X \quad$ Mn1 $\quad Y$

Kappa1, kappa2 1.00

dipoles $-.088 \quad 0.042-.032$

$\begin{array}{lllllll}\text { quadrupoles } & -.127 & 0.004 & -.025 & 0.002 & -.005\end{array}$

$\begin{array}{lllllllll}\text { octupoles } & 0.016 & -.027 & -.017 & -.029 & -.012 & 0.019 & -.014\end{array}$

\section{atom: 04}

\begin{tabular}{lll} 
Coordinate system Co1 & Z & \multicolumn{1}{c}{ C5 } \\
Kappa1, kappa2 1.011114 & 1.263355
\end{tabular}

Pv, P00 6.0540.

dipoles 0.00 .013

quadrupoles 0.1150 .013

atom: 05

coordinate system

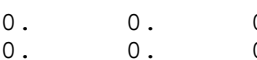

\begin{tabular}{lll} 
system Co1 & Z & \multicolumn{1}{c}{ C6 } \\
ppa2 1.011114 & 1.263355
\end{tabular}

Pv, P00 6.0840 .

dipoles 0 .

0.004

quadrupoles

octupoles

$\begin{array}{lll}0 . & 0 . & 0\end{array}$ atom: 06

$\begin{array}{llllllllllllll} & \mathrm{CO} & \mathrm{Z} & \mathrm{C} 4 & \mathrm{Y}\end{array}$

Kappa1, kappa2 1.011114 1.263355

Pv, P00 6.1150

$\begin{array}{lll}\text { dipoles } 0 . & 0 . & 0.029 \\ \text { quadrupoles } & 0.110 & 0 .\end{array}$

octupoles

$\begin{array}{cccc}-.023 & 0 . & 0 . & 0 \\ & & 0 & 0\end{array}$

atom: 07

\begin{tabular}{llll} 
Coordinate system Co2 & Z & \multicolumn{1}{c}{ C8 } & Y \\
Kappa1, kappa2 1.011114 & 1.263355 &
\end{tabular}

Pv, P00 6.101 0.

$\begin{array}{lll}\text { dipoles } 0 . & 0 . & 0.029\end{array}$

quadrupoles $\begin{array}{llll}0.087 & 0 . & 0 .\end{array}$

atom: 08

coordinate system

$\begin{array}{lllll}C 02 & Z & C 9 & Y\end{array}$

Kappa1, kappa2 1.011114 1.263355

PV, P00 6.1450.

dipoles 0.1450 .023

quadrupoles 0.1260.

atom: 09

coordinate system Co2

Pv, P00 6.147 0.

dipoles $0 . \quad 0.0 .032$

$\begin{array}{lll}0.132 & 0.032\end{array}$

octupoles

$\begin{array}{lll}.132 & 0 \\ -.010 & 0\end{array}$

atom: $\quad$ Mn 1

coordinate syst

Kappal, kappa2 0

dipoles $0.019 \quad-.175 \quad 0.022$

$\begin{array}{llllll}\text { quadrupoles } & 0.113 & 0.017 & -.122 & -.059 & -.033\end{array}$

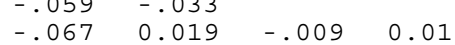

$\begin{array}{llllllll}\text { hexadecapoles } & -.005 & -.058 & 0.012 & -.094 & 0.101 & -.079 & 0.035\end{array}$

atom: Co1

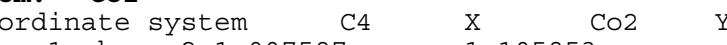

.

$0.043 \quad 0.010$

$\begin{array}{llllll}\text { quadrupoles } & 0.036 & 0.057 & 0.063 & -.038 & 0.004\end{array}$

$\begin{array}{llllllll}\text { octupoles } & -.012 & -.002 & -.034 & -.002 & 0.013 & -.018 & 0.019 \\ \text { hexadecapoles } & 0.108 & 0.056 & -.001 & 0.195 & -.073 & -.061 & 0.001\end{array}$ 


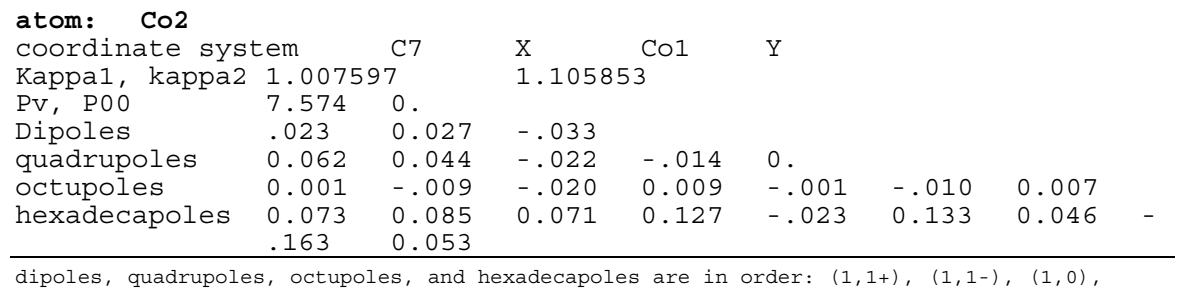

dipoles, quadrupoles, octupoles, and hexadecapoles are in order: $(1,1+),(1,1-),(1,0)$,

)$,(4,0),(4,1+),(4,1-),(4,2+),(4,2-),(4,3+),(4,3-),(4,4+),(4,4-)$ 
Table S6 Topological properties of the experimental and theoretical (italic) electron density at selected bond critical points. ${ }^{a}$

\begin{tabular}{|c|c|c|c|c|c|c|c|c|c|}
\hline atom1 & atom2 & d1 & d2 & $\lambda_{1}$ & $\lambda_{2}$ & $\lambda_{3}$ & $\nabla^{2} \rho(\mathrm{r})$ & $\rho(\mathrm{r})$ & $\varepsilon$ \\
\hline Mn1 & $\mathrm{C} 1$ & 0.884 & 0.910 & $\begin{array}{l}21.7 \\
20.65\end{array}$ & $\begin{array}{l}-4.6 \\
-4.05\end{array}$ & $\begin{array}{l}-4.8 \\
-3.86\end{array}$ & $\begin{array}{l}12.25 \\
12.74\end{array}$ & $\begin{array}{l}1.03 \\
1.03\end{array}$ & $\begin{array}{l}0.04 \\
0.05\end{array}$ \\
\hline Mn1 & $\mathrm{C} 2$ & 0.876 & 0.942 & $\begin{array}{c}21.3 \\
19.66\end{array}$ & $\begin{array}{l}-4.4 \\
-4.12\end{array}$ & $\begin{array}{l}-4.8 \\
-3.61\end{array}$ & $\begin{array}{l}12.11 \\
11.93\end{array}$ & $\begin{array}{l}0.97 \\
0.99\end{array}$ & $\begin{array}{l}0.07 \\
0.12\end{array}$ \\
\hline Mn1 & $\mathrm{C} 3$ & 0.927 & 1.033 & $\begin{array}{l}13.9 \\
11.57\end{array}$ & $\begin{array}{l}-3.2 \\
-3.83\end{array}$ & $\begin{array}{l}-3.5 \\
-3.25\end{array}$ & $\begin{array}{l}7.24 \\
4.49\end{array}$ & $\begin{array}{l}0.72 \\
0.82\end{array}$ & $\begin{array}{l}0.09 \\
0.15\end{array}$ \\
\hline $\mathrm{C} 1$ & 01 & 0.391 & 0.767 & $\begin{array}{l}66.5 \\
61.11\end{array}$ & $\begin{array}{l}-31.6 \\
-31.88\end{array}$ & $\begin{array}{l}-31.6 \\
-31.81\end{array}$ & $\begin{array}{l}3.22 \\
-2.58\end{array}$ & $\begin{array}{l}3.22 \\
3.31\end{array}$ & $\begin{array}{l}0.00 \\
0.0 \odot\end{array}$ \\
\hline C2 & 02 & 0.390 & $\odot .759$ & $\begin{array}{l}66.8 \\
63.77\end{array}$ & $\begin{array}{l}-34.2 \\
-32.73\end{array}$ & $\begin{array}{l}-33.6 \\
-32.62\end{array}$ & $\begin{array}{l}-0.94 \\
-1.58\end{array}$ & $\begin{array}{l}3.31 \\
3.36\end{array}$ & $\begin{array}{l}0.01 \\
0.00\end{array}$ \\
\hline C3 & 03 & 0.407 & 0.796 & $\begin{array}{l}41.7 \\
40.03\end{array}$ & $\begin{array}{l}-24.2 \\
-24.32\end{array}$ & $\begin{array}{l}-25.5 \\
-24.05\end{array}$ & $\begin{array}{l}-8.00 \\
-8.34\end{array}$ & $\begin{array}{l}2.91 \\
2.95\end{array}$ & $\begin{array}{l}0.05 \\
0.01\end{array}$ \\
\hline C3 & $\mathrm{C} 10$ & 0.738 & $\odot .702$ & $\begin{array}{c}11.3 \\
13.68\end{array}$ & $\begin{array}{l}-11.6 \\
-10.07\end{array}$ & $\begin{array}{l}-12.9 \\
-12.09\end{array}$ & $\begin{array}{l}-13.22 \\
-8.49\end{array}$ & $\begin{array}{l}1.86 \\
2.00\end{array}$ & $\begin{array}{l}0.10 \\
0.17\end{array}$ \\
\hline $\mathrm{C} 10$ & C31 & 0.747 & 0.750 & $\begin{array}{c}12.5 \\
10.84\end{array}$ & $\begin{array}{l}-11.1 \\
-9.40\end{array}$ & $\begin{array}{l}-12.1 \\
-11.47\end{array}$ & $\begin{array}{l}-10.74 \\
-10.47\end{array}$ & $\begin{array}{l}1.73 \\
1.77\end{array}$ & $\begin{array}{l}0.08 \\
0.18\end{array}$ \\
\hline C11 & C12 & 0.686 & 0.672 & $\begin{array}{c}11.6 \\
9.42\end{array}$ & $\begin{array}{l}-12.9 \\
-13.74\end{array}$ & $\begin{array}{l}-14.0 \\
-13.28\end{array}$ & $\begin{array}{l}-15.31 \\
-17.60\end{array}$ & $\begin{array}{l}2.21 \\
2.25\end{array}$ & $\begin{array}{l}0.08 \\
0.03\end{array}$ \\
\hline C12 & C41 & 0.749 & 0.719 & $\begin{array}{c}12.1 \\
9.42\end{array}$ & $\begin{array}{l}-11.5 \\
-12.17\end{array}$ & $\begin{array}{l}-12.3 \\
-11.52\end{array}$ & $\begin{array}{l}-11.66 \\
-14.27\end{array}$ & $\begin{array}{l}1.82 \\
1.86\end{array}$ & $\begin{array}{l}0.08 \\
0.05\end{array}$ \\
\hline Co1 & C11 & 1.009 & 1.029 & $\begin{array}{c}11.1 \\
10.15\end{array}$ & $\begin{array}{l}-0.5 \\
-2.58\end{array}$ & $\begin{array}{l}-2.5 \\
-0.78\end{array}$ & $\begin{array}{l}8.18 \\
6.79\end{array}$ & $\begin{array}{l}0.60 \\
0.69\end{array}$ & $\begin{array}{l}0.80 \\
0.70\end{array}$ \\
\hline Co1 & $\mathrm{C} 12$ & 0.972 & 1.020 & $\begin{array}{l}11.6 \\
10.69\end{array}$ & $\begin{array}{l}-0.9 \\
-2.77\end{array}$ & $\begin{array}{l}-2.5 \\
-1.83\end{array}$ & $\begin{array}{l}8.20 \\
6.09\end{array}$ & $\begin{array}{l}0.62 \\
0.73\end{array}$ & $\begin{array}{l}0.64 \\
0.73\end{array}$ \\
\hline Co2 & C11 & 0.962 & 1.003 & $\begin{array}{c}12.9 \\
11.40\end{array}$ & $\begin{array}{l}-1.1 \\
-3.03\end{array}$ & $\begin{array}{l}-3.0 \\
-2.24\end{array}$ & $\begin{array}{l}8.89 \\
6.13\end{array}$ & $\begin{array}{l}0.68 \\
0.77\end{array}$ & $\begin{array}{l}0.64 \\
0.26\end{array}$ \\
\hline $\mathrm{Co} 2$ & $\mathrm{C} 12$ & 0.970 & 1.005 & $\begin{array}{l}12.8 \\
10.69\end{array}$ & $\begin{array}{l}-1.0 \\
-2.77\end{array}$ & $\begin{array}{l}-2.9 \\
-1.83\end{array}$ & $\begin{array}{l}8.86 \\
6.09\end{array}$ & $\begin{array}{l}0.69 \\
\odot .76\end{array}$ & $\begin{array}{l}0.66 \\
0.34\end{array}$ \\
\hline Co1 & $\mathrm{C} 4$ & 0.873 & 0.925 & $\begin{array}{l}21.4 \\
20.45\end{array}$ & $\begin{array}{l}-4.7 \\
-3.78\end{array}$ & $\begin{array}{l}-4.6 \\
-3.83\end{array}$ & $\begin{array}{l}12.07 \\
12.84\end{array}$ & $\begin{array}{l}0.94 \\
0.98\end{array}$ & $\begin{array}{l}0.01 \\
0.02\end{array}$ \\
\hline Co1 & $\mathrm{C} 5$ & 0.893 & 0.938 & $\begin{array}{l}20.6 \\
19.66\end{array}$ & $\begin{array}{l}-4.6 \\
-4.65\end{array}$ & $\begin{array}{l}-4.5 \\
-3.71\end{array}$ & $\begin{array}{l}11.49 \\
11.30\end{array}$ & $\begin{array}{l}0.91 \\
0.92\end{array}$ & $\begin{array}{l}0.01 \\
0.20\end{array}$ \\
\hline Co1 & $\mathrm{c} 6$ & 0.894 & 0.934 & $\begin{array}{l}20.1 \\
19.55\end{array}$ & $\begin{array}{l}-4.4 \\
-3.71\end{array}$ & $\begin{array}{l}-4.4 \\
-3.51\end{array}$ & $\begin{array}{l}11.27 \\
12.33\end{array}$ & $\begin{array}{l}0.90 \\
0.92\end{array}$ & $\begin{array}{l}\odot .0 \odot \\
0.05\end{array}$ \\
\hline $\mathrm{C} 4$ & 04 & 0.407 & $\odot .739$ & $\begin{array}{l}46.4 \\
65.54\end{array}$ & $\begin{array}{l}-36.0 \\
-33.35\end{array}$ & $\begin{array}{l}-36.0 \\
-33.32\end{array}$ & $\begin{array}{l}-26.69 \\
-1.13\end{array}$ & $\begin{array}{l}3.49 \\
3.39\end{array}$ & $\begin{array}{l}\odot . \odot \odot \\
\odot . \odot \odot\end{array}$ \\
\hline C5 & 05 & 0.399 & $\odot .740$ & $\begin{array}{l}55.8 \\
68.68\end{array}$ & $\begin{array}{l}-35.9 \\
-35.98\end{array}$ & $\begin{array}{l}-35.9 \\
-34.70\end{array}$ & $\begin{array}{l}-16.04 \\
-2.00\end{array}$ & $\begin{array}{l}3.49 \\
3.44\end{array}$ & $\begin{array}{l}\odot .0 \odot \\
0.04\end{array}$ \\
\hline $\mathrm{C} 6$ & 06 & 0.403 & $\odot .740$ & $\begin{array}{l}51.5 \\
67.96\end{array}$ & $\begin{array}{l}-33.3 \\
-34.46\end{array}$ & $\begin{array}{l}-33.3 \\
-34.36\end{array}$ & $\begin{array}{l}-14.99 \\
-0.86\end{array}$ & $\begin{array}{l}3.38 \\
3.43\end{array}$ & $\begin{array}{l}\odot .0 \odot \\
0.0 \odot\end{array}$ \\
\hline $\mathrm{Co} 2$ & $\mathrm{C} 7$ & 0.880 & 0.924 & $\begin{array}{l}21.4 \\
20.26\end{array}$ & $\begin{array}{l}-4.7 \\
-3.78\end{array}$ & $\begin{array}{l}-4.6 \\
-3.74\end{array}$ & $\begin{array}{l}12.07 \\
12.74\end{array}$ & $\begin{array}{l}0.95 \\
0.97\end{array}$ & $\begin{array}{l}0.02 \\
0.01\end{array}$ \\
\hline $\mathrm{Co} 2$ & $\mathrm{C} 8$ & 0.898 & 0.936 & $\begin{array}{l}20.4 \\
19.47\end{array}$ & $\begin{array}{l}-4.5 \\
-3.66\end{array}$ & $\begin{array}{l}-4.6 \\
-3.49\end{array}$ & $\begin{array}{l}11.31 \\
12.32\end{array}$ & $\begin{array}{l}0.91 \\
0.91\end{array}$ & $\begin{array}{l}0.01 \\
0.05\end{array}$ \\
\hline
\end{tabular}


Table S6 (continued)

\begin{tabular}{llllllllll} 
C02 & C9 & 0.884 & 0.934 & 20.7 & -4.8 & -4.6 & 11.29 & 0.93 & 0.03 \\
& & & & 20.07 & -5.71 & -3.78 & 10.58 & 0.94 & 0.34 \\
C7 & \multirow{2}{*}{07} & 0.404 & 0.739 & 48.8 & -35.3 & -35.3 & -21.82 & 3.52 & 0.00 \\
& & & & 67.38 & 33.91 & 33.88 & -0.41 & 3.42 & 0.00 \\
C8 & \multirow{2}{*}{08} & \multirow{2}{*}{0.400} & 0.739 & 54.9 & -35.0 & -35.0 & -15.12 & 3.48 & 0.00 \\
& & & & 69.04 & -34.70 & -34.68 & -0.34 & 3.45 & 0.00 \\
C9 & 09 & \multirow{2}{*}{0.400} & 0.742 & 55.8 & -34.8 & -34.8 & -13.89 & 3.45 & 0.00 \\
& & & & 68.13 & -36.77 & -34.44 & -3.08 & 3.43 & 0.06
\end{tabular}

$\bar{a} \mathrm{~d} 1$ and $\mathrm{d} 2$ are the distances $(\AA)$ from the $b c p$ to each of the linked atoms, respectively; $\rho(\mathrm{r})$, $\nabla^{2} \rho(\mathrm{r})$, and $\varepsilon$ are the ED $\left(\mathrm{e} . \AA^{-3}\right)$, the laplacian of the ED (e. $\left.\AA^{-5}\right)$, the ellipticity of the bond. 
$0.71<d<7.69(A)$

$\mathrm{MIN}=-0.2332 \quad \mathrm{MAX}=0.1615$

$1 \mathrm{~A}$

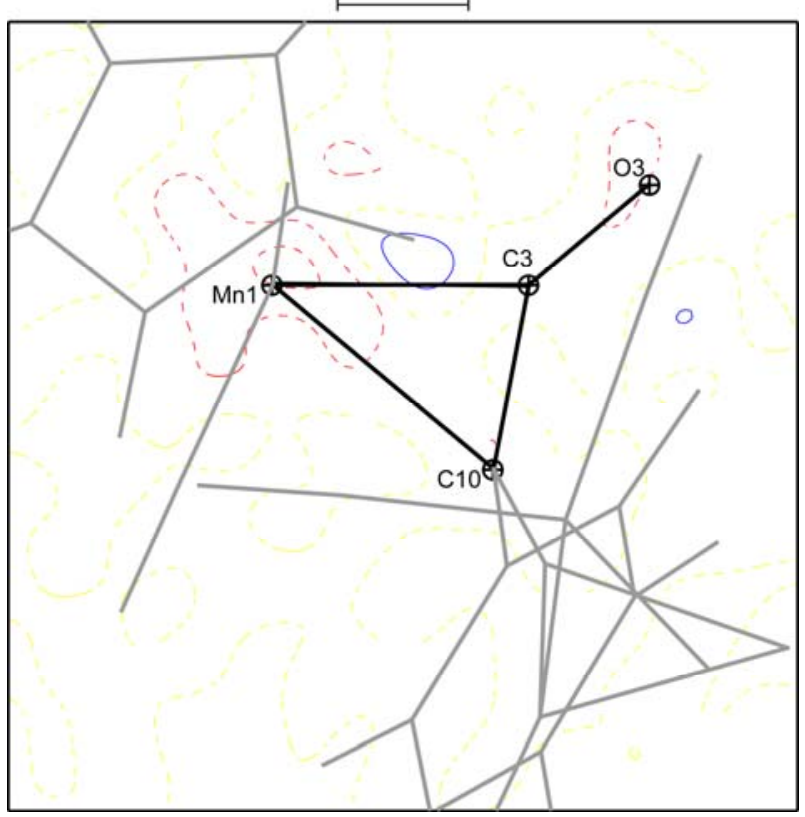

Fig. S1 Residual electron density in $\mathbf{1}$ after the multipolar refinement in the plane C10/ Mn1/C3 (0.1 e. $\AA^{-3}$ isocontours, $\mathrm{S}<0.7 \AA^{-1}$ ).

\section{$0.71<\mathrm{d}<7.69(\mathrm{~A})$}

MIN $=-0.4123 \quad$ MAX $=0.8791$

$1 \mathrm{~A}$

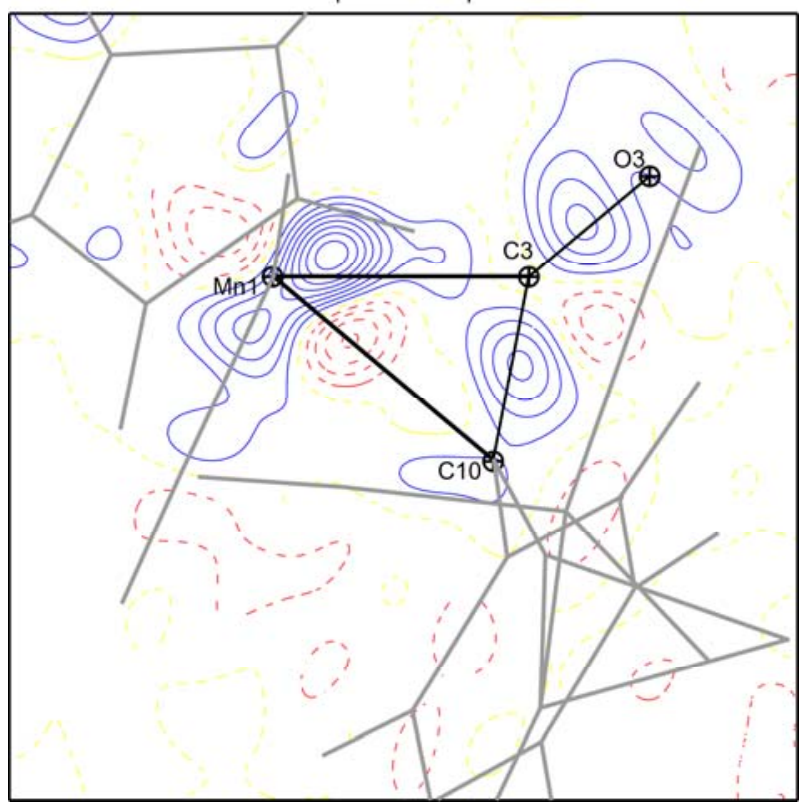

Fig. S2 Experimental deformation map for 1 in the plane C10/ Mn1/C3 (0.10 e. $\AA^{-3}$ isocontours, $\left.S<0.7 \AA^{-1}\right)$. 


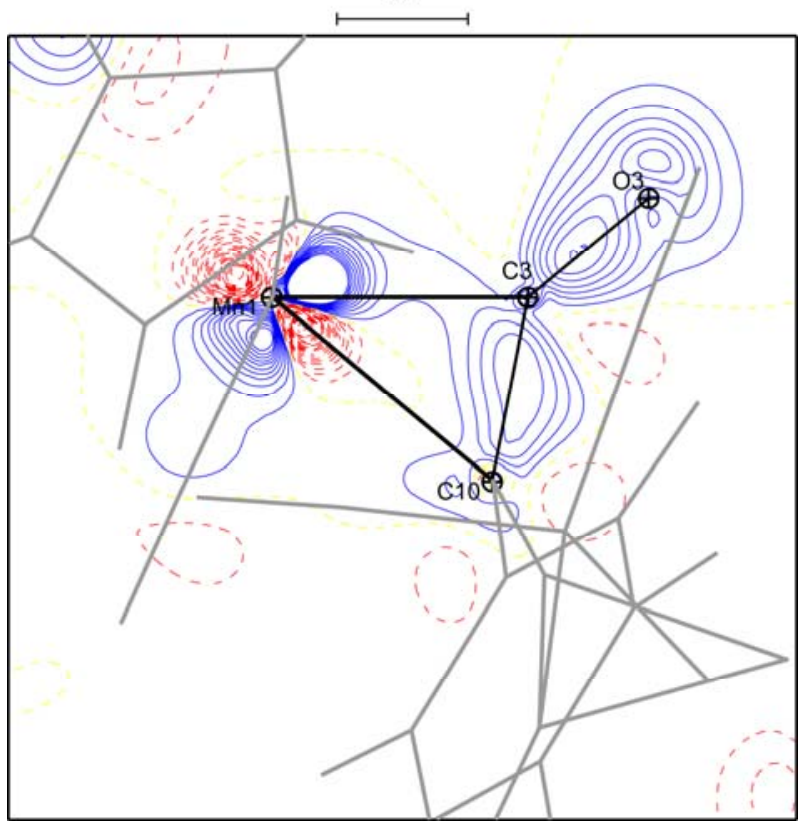

Fig. S3 Experimental static deformation map for 1 in the plane C10/ Mn1/C3 (0.10 e. $\AA^{-3}$ isocontours, $S<0.7 \AA^{-1}$ ) 


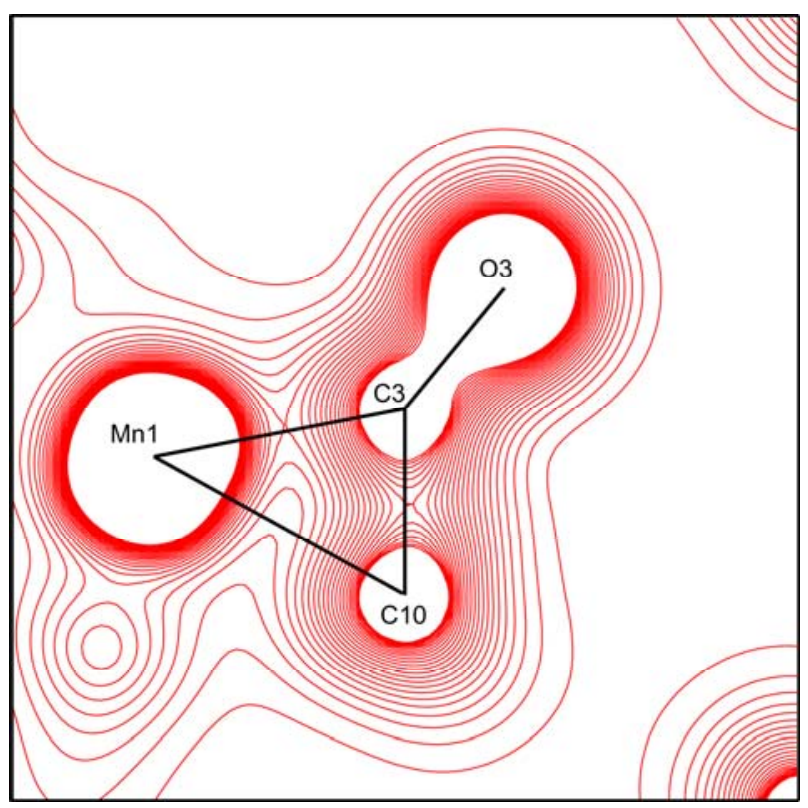

Fig. S4 Total experimental ED $\rho(\mathrm{r})$ for $\mathbf{1}$ in the Mn1/C3/C10 plane (0.1 e. $\AA^{-3}$ isocontours)

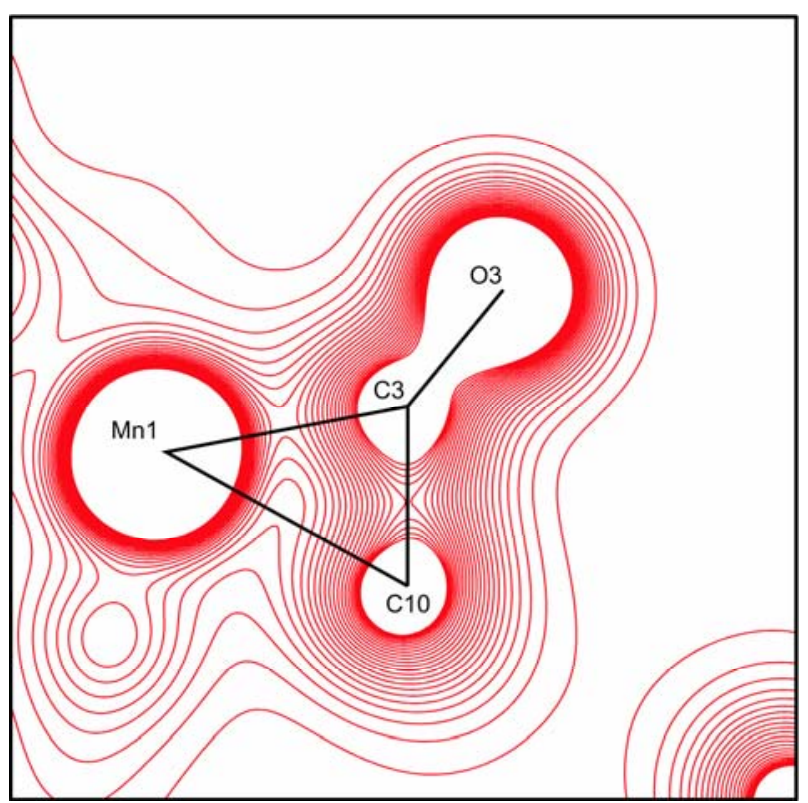

Fig. S5 Total theoretical ED $\rho(\mathrm{r})$ for $\mathbf{1}$ in the Mn1/C3/C10 plane (0.1 e. $\AA^{-3}$ isocontours) 


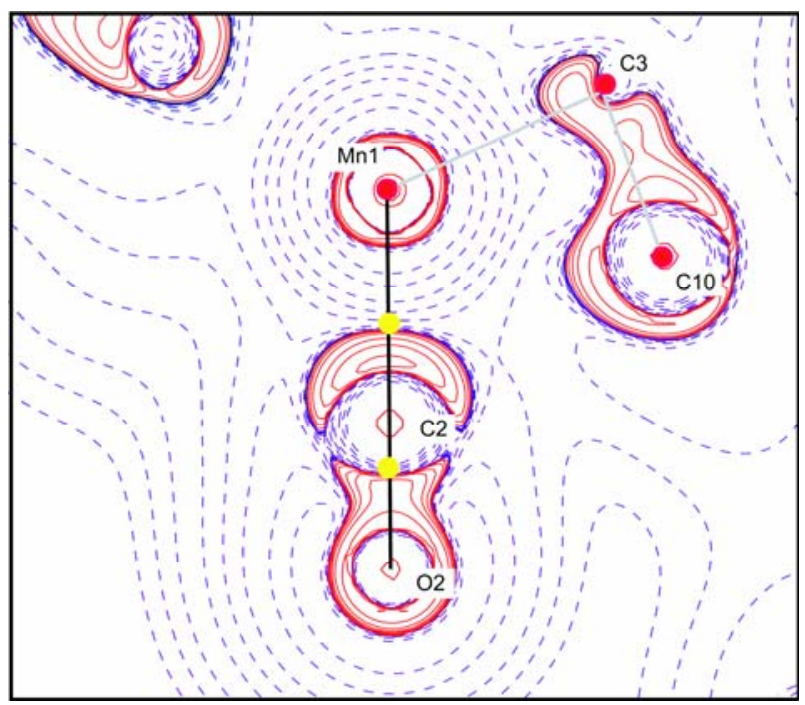

Fig. S6 Laplacian of the theoretical electron density for $\mathbf{1}$, in the $\mathrm{C} 2 / \mathrm{Mn} 1 / \mathrm{C} 3$ plane (contours are drawn at 0.000 , $\pm 2.0 \times 10 \mathrm{n}, \pm 4.0 \times 10 \mathrm{n}, \pm 8.0 \times 10 \mathrm{n}$ e. $\AA^{-5}$ levels; where $\mathrm{n}$ $=0,-3, \pm 2, \pm 1)$. 


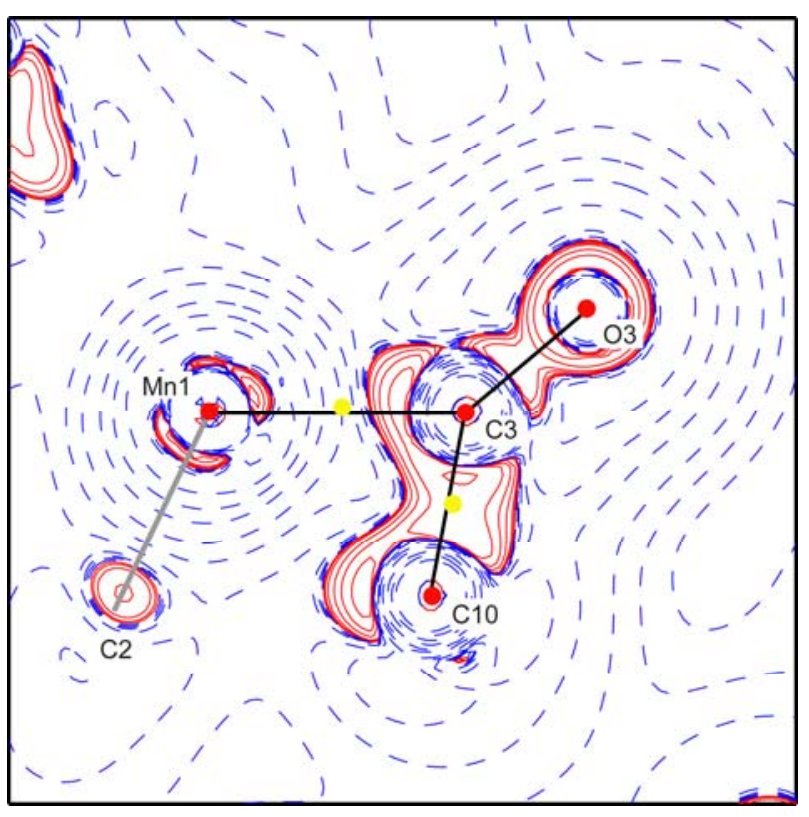

Fig. S7 Laplacian of the experimental electron density for $\mathbf{1}$, in the $\mathrm{C} 10 / \mathrm{Mn} 1 / \mathrm{C} 3$ plane (contours are drawn at $0.000, \pm 2.0 \times 10 \mathrm{n}, \pm 4.0 \times 10 \mathrm{n}, \pm 8.0 \times 10 \mathrm{n}$ e. $\AA^{-5}$ levels; where $n=0,-3, \pm 2, \pm 1$ ).

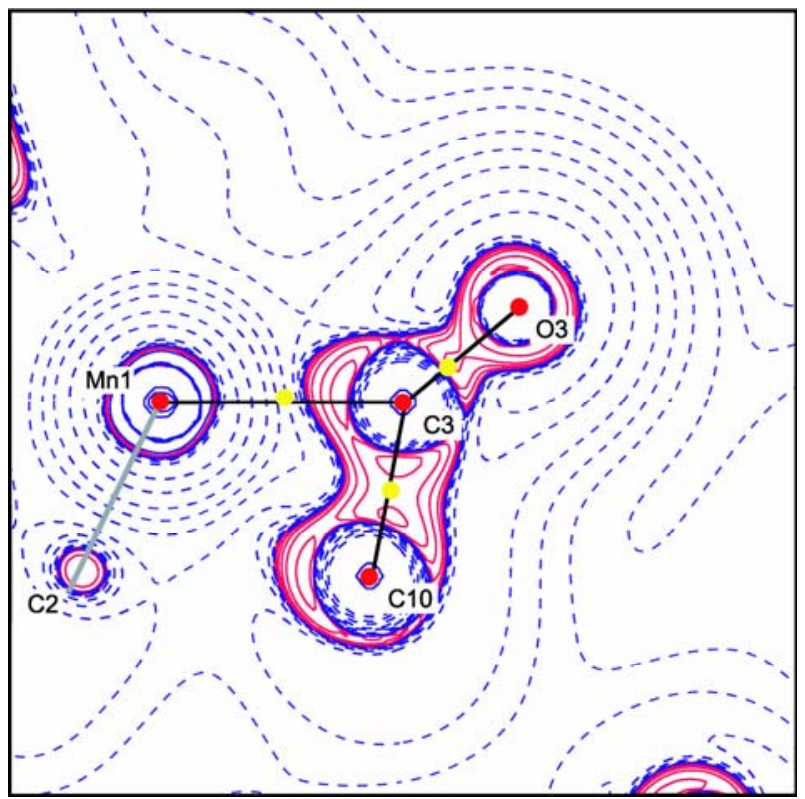

Fig. S8 Laplacian of the theoretical electron density for $\mathbf{1}$, in the $\mathrm{C} 10 / \mathrm{Mn} 1 / \mathrm{C} 3$ plane (contours are drawn at $0.000, \pm 2.0 \times 10 \mathrm{n}, \pm 4.0 \times 10 \mathrm{n}, \pm 8.0 \times 10 \mathrm{n}$ e. $\AA^{-5}$ levels; where $n=0,-3, \pm 2, \pm 1$ ). 